\title{
Design, Fabrication, and Critical Current Testing of No-Insulation Superconducting Rotor Coils for NASA's 1.4 MW High-Efficiency Megawatt Motor
}

\author{
Dr. Justin J. Scheidler* and Thomas F. Tallerico ${ }^{\dagger}$ \\ NASA Glenn Research Center, Cleveland, Ohio, 44135
}

\begin{abstract}
NASA Glenn Research Center is developing a 1.4 MW high-efficiency electric machine for future electrified aircraft to reduce energy consumption, emissions, and noise. This wound-field, synchronous machine employs a self-cooled, superconducting rotor to achieve excellent specific power and efficiency. This paper discusses the design and fabrication of the no-insulation high temperature superconducting (HTS) rotor coils and compares them to conventionally insulated HTS coils. Two sub-scale test coils with epoxy on only one axial face were fabricated. Critical current testing of the coils at $77 \mathrm{~K}$ and self field was conducted to study the influence of thermal cycling on their critical current and n-value. After two or four aggressive thermal cycles between $77 \mathrm{~K}$ and about $278 \mathrm{~K}\left(5^{\circ} \mathrm{C}\right)$, the critical current and n-value were nearly unchanged, indicating very little to no degradation.
\end{abstract}

\section{Introduction}

$T^{O}$ drastically reduce the energy consumption, emissions, and noise of future commercial transport aircraft, NASA is investing in a broad portfolio of enabling research and development for electrified aircraft propulsion (EAP) [1]. A number of transport aircraft concepts that harness EAP have been shown to provide system-level benefits to energy consumption, fuel burn, and/or emissions [2-8]. As expected, the existence of the benefits and their magnitude depend on the assumed performance of the individual EAP components. Two key performance parameters that have been identified for enabling single aisle aircraft with EAP are the specific power and efficiency of the EAP system's electric machines. Specific power several times greater than industrial motors and efficiency at or above the state of the art (96\%) are typically needed for an aircraft concept to close with a benefit to one or more of the aforementioned performance metrics. Accordingly, NASA is investing in the development of three separate megawatt-class electric machines that combine high specific power $(>13 \mathrm{~kW} / \mathrm{kg})$ with high efficiency $(>96 \%)$ [1]. One of these machines is being developed at NASA's Glenn Research Center. NASA's machine, referred to as the High-Efficiency Megawatt Machine (HEMM), is a 1.4 MW wound field synchronous machine that is designed to be $>98 \%$ efficient with $>16 \mathrm{~kW} / \mathrm{kg}$ specific power. To illustrate the effect of this machine on a transport aircraft with EAP, system-level studies have been performed for one concept aircraft, the NASA single-aisle turboelectric aircraft with aft boundary layer propulsor (STARC-ABL). Relative to the baseline STARC-ABL configuration, the electric machine performance targets established by NASA (13 kW/kg, $96 \%$ ) provide a $7 \%$ to $10 \%$ reduction in fuel burn [7]. Compared to this benefit of NASA's performance targets, the more stringent goals of HEMM (16 kW/kg, 98\% to 99\%) provide an additional $1.5 \%$ to $2.5 \%$ reduction in fuel burn, respectively [1]. It should be noted that further refinements to the analyses in [7] (and referenced in [1]) are currently being conducted and updated results are expected soon. A potentially more impactful benefit is that such an increase in efficiency will reduce the amount of waste heat that must be managed by a factor of 2 to 4 , respectively.

The exceptional performance of NASA's HEMM is primarily achieved by utilizing a semi-slotless stator and an internally-cryocooled, superconducting rotor. Superconducting field windings provide unmatched magnetic field strengths and a negligible amount of internal energy loss, but they introduce a few challenges. Superconductors must be kept at cryogenic temperatures and the superconducting system must be carefully designed to avoid a loss of superconductivity, which will occur when the temperature, magnetic flux density, or conduction current in the superconductor exceed design limits. Additionally, superconductors are fragile compared to copper and aluminum conductors; superconductors are subject to maximum strain and minimum bend radius constraints, and care must be taken to avoid appreciable shear stresses or excessive tensile stresses. These mechanical constraints are particularly important for superconducting rotor coils, which are subjected to significant centrifugal loads in addition to the typical

\footnotetext{
*Research Mechanical Engineer, Rotating and Drive Systems Branch, 21000 Brookpark Road, Cleveland, Ohio, 44135.

${ }^{\dagger}$ Research Engineer, Rotating and Drive Systems Branch, 21000 Brookpark Road, Cleveland, Ohio, 44135.
} 
thermal and electromagnetic forces. Also, due to their high cost, it is important to carefully design the system and conduct sub-scale testing before full-scale implementation to sufficiently reduce the risks imposed by the aforementioned challenges.

This paper discusses the design and fabrication of high temperature superconducting (HTS) rotor coils without turn-to-turn electrical insulation for NASA's HEMM. First, the design of HEMM's rotor is summarized to provide context for the remainder of the paper. Then, the characteristics of the coils, the motivation for using uninsulated coils, and important design considerations are discussed. A few sub-scale HTS coils were fabricated using a new 3D printed winding fixture. The key features of the test coils and winding fixture are described. Finally, the ability of the coil's design to resist degradation from thermal cycling is assessed by measuring the critical current and n-value of two sub-scale coils at $77 \mathrm{~K}$ and self field before and after thermal cycling.

\section{Rotor Design}

To put the coil design in perspective, the design of HEMM's rotor is first summarized. Table 1 lists the key characteristics and performance goals of HEMM, whereas Table 2 lists the characteristics of the rotor. The magnetomotive force of each rotor coil is nearly 100 times greater than that of each copper stator coil, which are operated above room temperature. Consequently, the time-varying magnetic flux density $(B)$ in the HTS rotor coils is negligible $(<0.006 \mathrm{~T})$. Also, the rotor coils are excited with only a DC current. Thus, AC losses in the rotor coils are negligible and a HTS tape conductor can be utilized. Although the rotor's tip speed $(107 \mathrm{~m} / \mathrm{s})$ is relatively low for an electric machine, the centrifugal force imposed on each rotor coil is still significant considering that superconductors are much more fragile than copper wire and the space for the containment structure is limited (i.e., the pole count is fairly high). As a result, the rotor coils must be carefully designed and constructed so that the centrifugal force imposed on them is readily transferred to the containment structure. For further details about the design of HEMM and its rotor, see Jansen et al. [9] and Scheidler [10].

Table 1 Key characteristics and performance goals of NASA's High Efficiency Megawatt Machine (HEMM).

\begin{tabular}{clccc}
\hline $\begin{array}{l}\text { Continuous power } \\
\text { rating, MW }\end{array}$ & Motor type & $\begin{array}{l}\text { Speed, } \\
\text { rpm }\end{array}$ & $\begin{array}{l}\text { Specific power } \\
\text { goal, kW/kg }\end{array}$ & $\begin{array}{l}\text { Efficiency } \\
\text { goal, \% }\end{array}$ \\
\hline 1.4 & Wound field synchronous & 6,800 & 16 & $>98$ \\
\hline
\end{tabular}

Table 2 Characteristics of the rotor for NASA's High Efficiency Megawatt Machine (HEMM).

\begin{tabular}{rcrc}
\hline Characteristic/parameter & Value & Characteristic/parameter & Value \\
\hline Electrical frequency, $\mathrm{Hz}$ & $\mathrm{DC}$ & Outside diameter, cm & 30 \\
Number of poles & 12 & Inside diameter, cm & 20.5 \\
Material & solid Fe $_{49.15} \mathrm{Co}_{48.75} \mathrm{~V}_{2}$ & Axial length, cm & 12.5 \\
\hline
\end{tabular}

\section{Coil Design}

The characteristics of the rotor coils for HEMM are summarized in Table 3 A 2nd generation HTS (REBCO: rare Earth-barium-copper-oxide) was selected over other superconductors, because they are commercially available in long piece lengths and can provide sufficient performance at high temperatures (about $77 \mathrm{~K}$ and below) in moderately strong magnetic environments, and, as mentioned above, $\mathrm{AC}$ losses are not a concern. The operating current and temperature were defined based on the manufacturer's measurements and de-rating factors for the $B$-orientation-dependence of the critical current and estimates of manufacturing variation and modeling inaccuracy [10]. Each rotor coil is composed of 4 racetrack coils connected together by soldered HTS joints. Due to the relatively high number of rotor poles and the desire to maximize the flux density produced by the rotor, the rotor coils have many turns and their minimum bend radius is at the limit specified by the manufacturer. 
Table 3 Characteristics of the rotor coils for NASA's High Efficiency Megawatt Machine (HEMM).

\begin{tabular}{rcrc}
\hline Characteristic/parameter & Value & Characteristic/parameter & Value \\
\hline Superconductor & $\begin{array}{c}\text { 2nd generation } \\
\text { REBCO } \\
\text { none }\end{array}$ & Superconductor width, mm & 4 \\
Superconductor thickness, $\mu \mathrm{m}$ & 65 \\
Expected critical current (at & Min. superconductor bend & 15 \\
$77 \mathrm{~K}$, self field), A & 150 & Nudius, mm & 4 \\
Max. magnetic flux density in & 2 & Number of turns per layer & 230 \\
the superconductor, T & 62.8 & & \\
Operating temperature, K & 51.5 & & \\
Operating current, A & & & \\
\hline
\end{tabular}

One of the challenges with using superconductors is quench, or a loss of superconductivity as a result of excessive temperature, magnetic flux density, or conduction current in the superconductor. Quench is particularly important in REBCO HTS, because the quenched zone grows slowly (on the order of $10^{-3}$ to $10^{-1} \mathrm{~m} / \mathrm{s}$ [11]). For some HTS coils, the temperature in the quenched zone can reach the melting temperature of copper within a fraction of a second [12]. Although this issue is mitigated by limiting the applied current to a fraction of the superconducting coil's critical current, quench may still occur due to a number of reasons, such as inaccuracies in the analysis, unexpected heat sources, excessive mechanical strain in the superconductor, or transients in the operating state. Consequently, controllers are often used to monitor the state of the coil and rapidly reduce the current when quench is detected. Another method of quench protection is to remove the turn-to-turn electrical insulation from the coil [13]; this method provides inherent quench protection by allowing the applied current to flow around a quenched zone in the superconductor. Other benefits of so-called no insulation coils include higher engineering current density, better mechanical strength, and resistance to defects in the superconductor. The improvement in engineering current density results from not only the lack of an insulation layer between turns but also because the conductor's copper encapsulation doesn't need to carry the full operating current during a quench event. Specifically, the superconductor's thickness can be reduced to $65 \mu \mathrm{m}$ (from the typical $100 \mu \mathrm{m}$ ), which for a given coil volume enables the coil to have a 54\% higher magnetomotive force. The downside of no insulation coils is that they exhibit a charging delay (i.e., the magnetic field produced by the coil lags in time behind the applied current). However, this is a minimal concern for the field winding of an electric machine, because the applied current is DC and the time constants of the charging delay are typically small (on the order of seconds or less) compared to even short flight missions. Therefore, no insulation HTS coils were selected for the rotor of HEMM.

Although they were suggested only recently, several papers have reported the fabrication and critical current testing of no insulation coils [13-15]. However, although they have been proposed for use in the rotor of an electric machine [16], no insulation rotor coils have not been demonstrated. The presence of significant centrifugal forces in the rotor of aircraft electric machines complicates the design of the no insulation coil and its mechanical support structure. To ensure that the coil mechanically responds as a whole and the self protection capability is maintained throughout operation, some form of overbanding [17], epoxy/adhesive, or other mechanical feature is needed; without this, the turn-to-turn contact will be lost and the turns will deform independently under the centrifugal and magnetic forces. This feature is also useful for maintaining the coil's shape during handling, soldering, and assembly. As a first attempt, the sub-scale practice coils shown in this paper will be bound together using a cryogenic epoxy applied to one of their axial faces.

For epoxy impregnated coils wound with HTS tapes, a cryogenic epoxy must be carefully selected, because thermal expansion mismatches between the epoxy and HTS commonly cause delamination of the thin film superconductor from its substrate during thermal cycling [18, 19]. The thermally-induced stresses and strains are more severe for non-circular coils with more turns and smaller radii [20], such as the coils for HEMM. However, delamination due to thermal cycling was not observed in dry-wound coils [18] (like some of the no insulation coils proposed here), because radial tensile stresses between turns cannot be supported. Nevertheless, care was taken to select an epoxy. In HEMM, thermal conductivity is also important, because the rotor coils are conductively cooled. Stycast 2850FT Black with catalyst 23LV was chosen for its combination of low thermal expansion, high thermal conductivity, and moderate viscosity.

The rotor of HEMM is conductively cooled by a cryocooler that has a $50 \mathrm{~K}$ cold head, which is less than $13 \mathrm{~K}$ below the max operating temperature of the HTS rotor coils. As a result, heat input to the rotor must be minimized, especially 
in or near the HTS coils. The resistance of the solder joints between the coil layers and both the current terminals and HTS layer-to-layer bridges must therefore be minimized. Non-pre-tinned solder ribbon was selected to provide the lowest and most reproducible joint resistivity [21, 22]. To stay well within the manufacturer's suggested soldering temperature limit of $200{ }^{\circ} \mathrm{C}$ while maintaining adequate strength, 52In $48 \mathrm{Sn}$ solder (melting temperature of $118{ }^{\circ} \mathrm{C}$ ) was chosen. If a multi-step soldering approach becomes necessary, $63 \mathrm{Sn} 37 \mathrm{~Pb}$ (melting temperature of $183{ }^{\circ} \mathrm{C}$ ) and/or 97In Ag3 (melting temperature of $143{ }^{\circ} \mathrm{C}$ ) will be used for the initial step(s).

\section{Coil Fabrication}

In order to mitigate risk and validate the rotor coil design, a few uninsulated HTS coils have been manufactured for critical current, thermal cycling, and rotational loading testing. Due to the high cost of HTS, these test coils where manufactured at a smaller scale than the final rotor coils. The test coils (Fig. 1) each have 25 turns and a reduced axial length relative to the final rotor coil design. The minimum bend radius in each coil matches that of the final rotor design. Straight sections where included both in the end turns and active sections of each coil, because stress analysis showed that the transition regions between the straight and curved sections contained the highest stress in the final rotor design. Sufficient length was included in the active section in order to allow room for multiple coils to be stacked and soldered together for future testing.

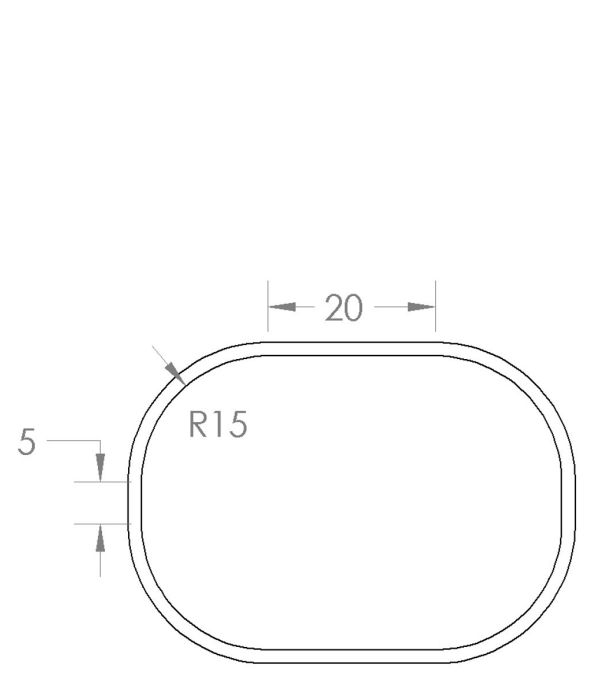

(a)

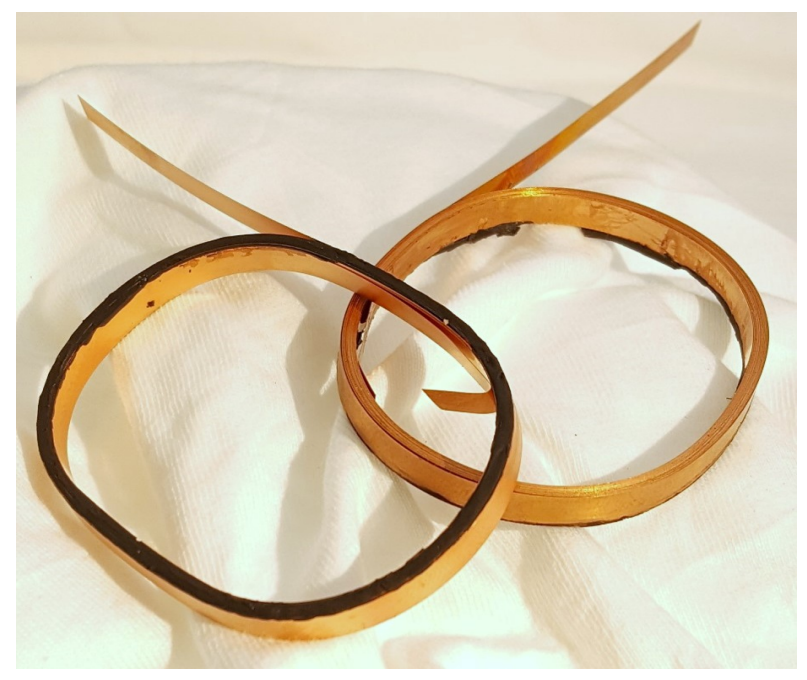

(b)

Fig. 1 Sub-scale high temperature superconducting coils for risk reduction testing of HEMM's rotor coils: (a) dimensions (in units of $\mathbf{~ m m}$ ) and (b) completed 25 turn coils with cryogenic epoxy applied to one axial face.

In order to fabricate these test coils, a 3D printed nylon winding fixture (Fig. 2) was designed. 3D printing was used to reduce manufacturing lead time and cost. It also allowed for multiple design iterations and continuous improvement of the winding fixture. The downside of this approach is that high temperature softening of the nylon may prevent the use of some solders while the coil is fixtured. The fixture consists of a baseplate, a tooth, two side clamps, and a cap. The baseplate is mounted to ground through a double row angular contact bearing for stability and ease of rotating. The tooth defines the shape of the coil. It is made as a separate part from the baseplate so that coils can be easily removed from the fixture. The two side clamps are used to set and hold the width of the coil while the epoxy cures. The coil's width is a critical dimension in the final rotor design. The side clamps are printed to an exact size for a given coil's number of turns, and a screw is used to lock in their position precisely to guarantee the correct width is achieved. Any excess length in the coils would be pushed into the end windings. The cap is used to make sure the coil's axial face is flat. When epoxy is used, it is first applied to the cap, then the cap is placed on the coil, and the whole assembly is inverted to sit on the cap. This inversion of the fixture reduces the amount of epoxy that ends up on the coil's radial faces, keeping them clean for solder joints. 


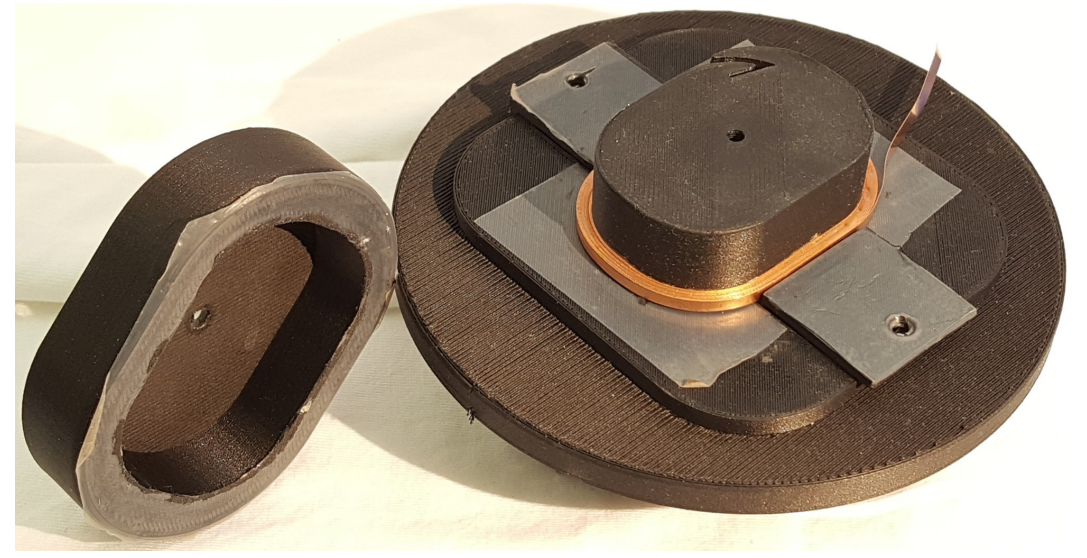

Fig. 2 3D printed coil winding fixture with practice copper coil wound in place.

\section{Critical Current Testing}

The critical current $I_{\mathrm{c}}$ and n-value $n$ of two sub-scale, uninsulated test coils were measured at $77 \mathrm{~K}$ and self field using the test rig depicted in Fig. 3. The key components of the rig are a dewar filled with liquid nitrogen, a linear amplifier capable of up to 120 A DC current, a nanovoltmeter, and a data acquisition system. During a test, the DC current was slowly stepped up in small increments and a measurement was recorded at each step after holding the current for $5 \mathrm{~s}$ or more. This hold duration was more than sufficient to overcome the charging delay of the uninsulated coil [23], which was $<1 \mathrm{~s}$. The voltage taps are located about $10 \mathrm{~cm}$ away from each current lead to ensure that the measured current-transfer voltage at the critical current is less than $0.01 \%$ of the expected voltage at the critical current (based on $1 \mu \mathrm{V} / \mathrm{cm}$ electric field criteria) [24]. As shown in Fig. 4, each coil was mounted to a G10 plate, which was suspended in the liquid nitrogen.

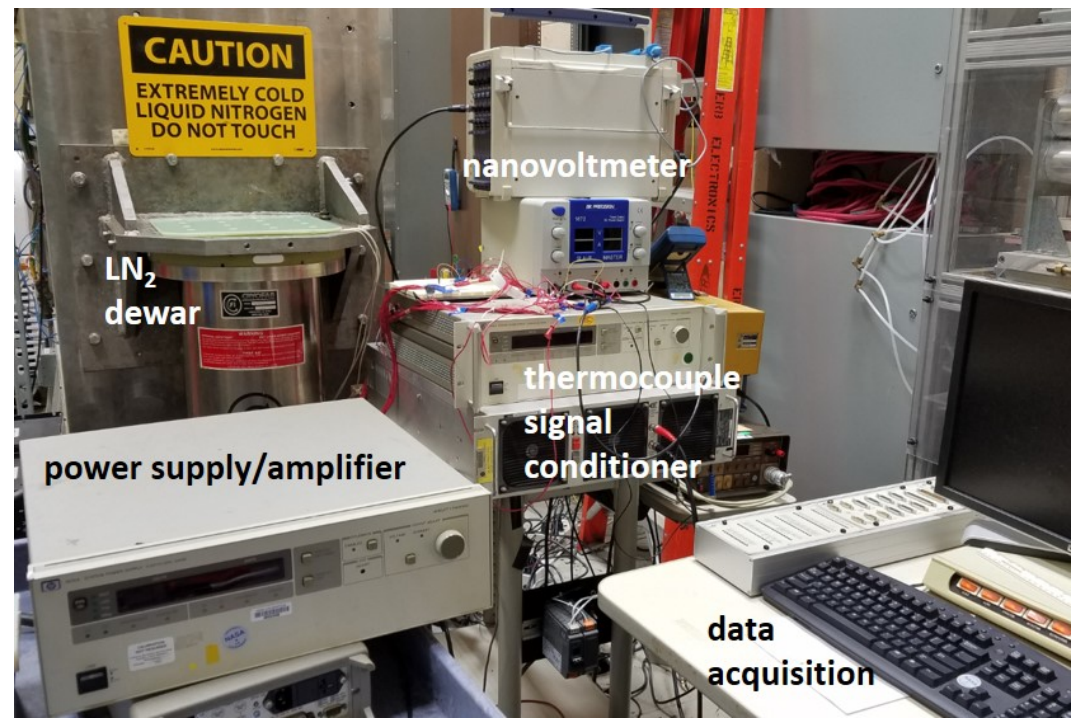

Fig. 3 NASA Glenn Research Center's high temperature superconducting coil test rig.

The purpose of the initial tests reported here was to demonstrate that the test coils described above could survive multiple aggressive thermal cycles. Each coil was tested twice. First, the coil was very slowly lowered into the liquid nitrogen bath to ensure a low thermal gradient. Voltage measurements were recorded while increasing and decreasing the applied DC current. The coil was then removed from the dewar and allowed to warm up to about $5{ }^{\circ} \mathrm{C}$. Then, the coil was rapidly inserted into the liquid nitrogen and allowed to reach a steady temperature. This process of coil removal and insertion was then repeated for a total of two thermal cycles for coil 1 and four for coil 2. After, the critical current measurements were repeated. An electric field criteria $(1 \mu \mathrm{V} / \mathrm{cm})$ was used to define the critical current. 


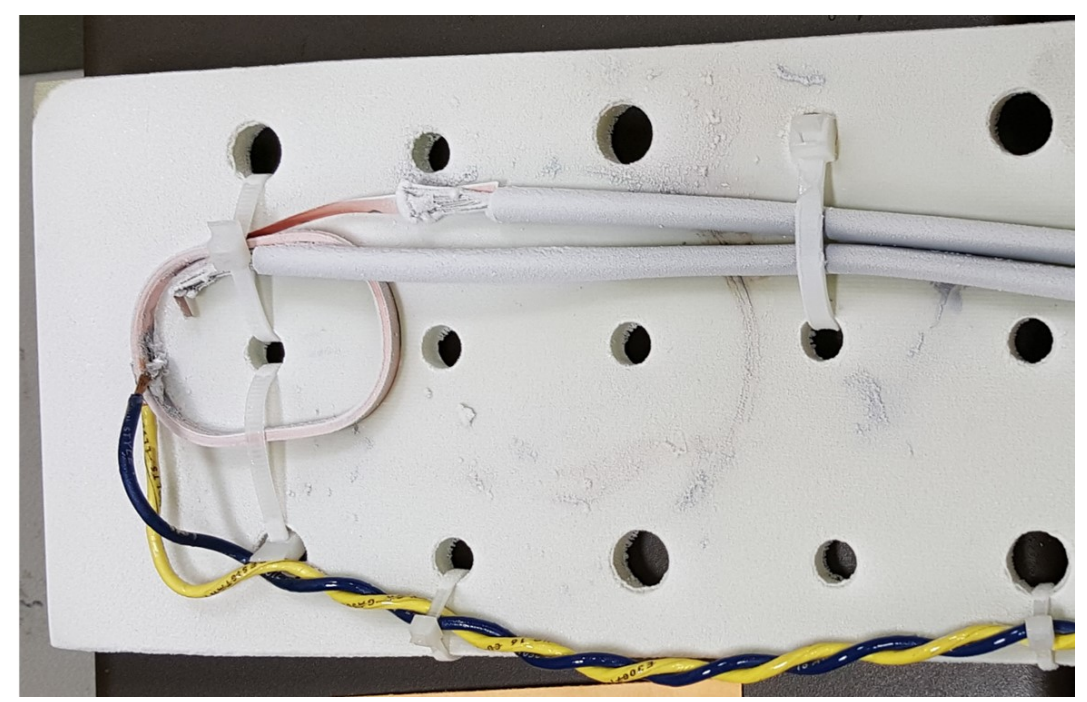

Fig. 4 Sub-scale HTS test coil mounted to a G10 plate, shown after testing in liquid nitrogen.

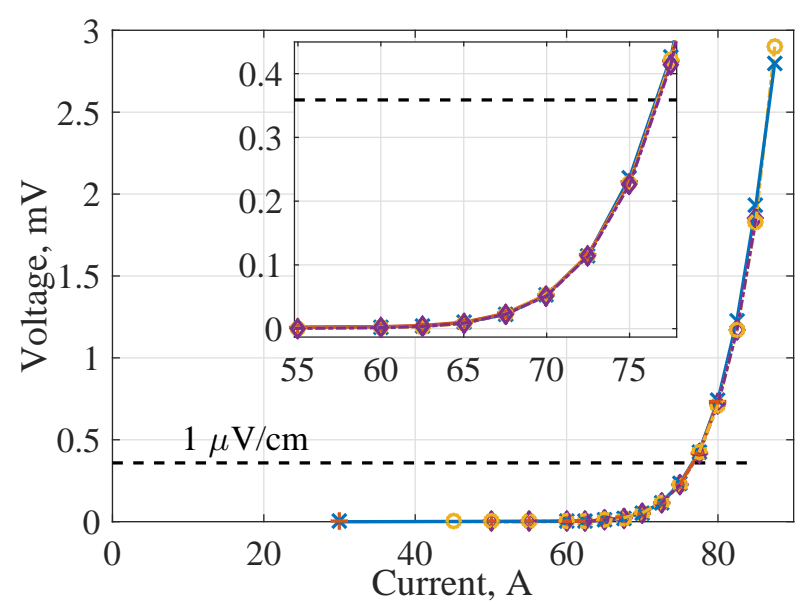

(a)

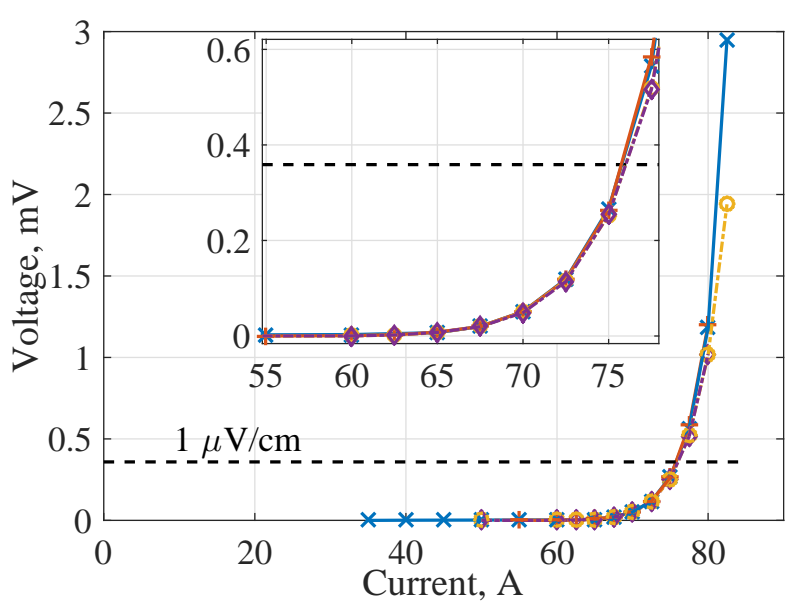

(b)

Fig. 5 Critical current measurements of two sub-scale HTS test coils (a) coil 1 and (b) coil 2; before thermal cycling (solid) and after thermal cycling (dash-dotted); the electric field criteria $(1 \mu \mathrm{V} / \mathrm{cm})$ used to define the critical current is indicated by the horizontal dashed line.

The voltage response of each coil for all 4 cases (increasing and decreasing current, before and after thermal cycling) is shown in Fig. 5. For these initial tests, the current was limited to about $1.15 I_{\mathrm{c}}$, although no insulation HTS coils in liquid nitrogen are capable of carrying $>4 I_{\mathrm{c}}$ without damage [15]. A high degree of repeatability is observed, indicating that thermal degradation is minimal or nonexistent. To further investigate this, the critical current and n-value of each curve was calculated by fitting the following function to the data using an unconstrained minimization algorithm,

$$
\frac{V}{V_{\mathrm{c}}}=\left(\frac{I}{I_{\mathrm{c}}}\right)^{n}
$$

where $V_{\mathrm{c}}$ is the expected voltage at the critical current; the results are tabulated in Table 4 . Except for the n-value in one instance, the performance metrics are consistent between increasing and decreasing current, which lends credence to the data. The critical current and n-value of coil 1 remain constant (at least to the measurement uncertainty) after two aggressive thermal cycles, indicating that thermal degradation did not occur. After coil 2 was thermally cycled four times, its n-value slightly decreased, suggesting the possibility of minor degradation, but its critical current slightly 
increased, providing a weak conflicting trend. Thus, the results for coil 2 are not conclusive, but at worst indicate only minor degradation of only the n-value. These results differ from findings by Shin et al. [16], who observed a $I_{\mathrm{c}}$ reduction of almost $2 \%$ in a similar uninsulated HTS coil after four thermal cycles between room temperature and $77 \mathrm{~K}$. The main differences between the two tests are that their coil is fully encapsulated with the epoxy on the coil's two exposed surfaces and the exterior of their impregnated coil may have been cooled a little slower. This may suggest that the degradation observed by Shin et al. [16] is caused by the presence of epoxy on the outer radial surface of the coil.

Table 4 Measured critical current $I_{\mathrm{c}}$ and n-value $n$ at self field and $77 \mathrm{~K}$ for two test coils.

\begin{tabular}{rcccc}
\hline & \multicolumn{2}{c}{ Coil 1 } & \multicolumn{2}{c}{ Coil 2} \\
Test & $I_{\mathrm{c}}, \mathrm{A}$ & $n$ & $I_{\mathrm{c}}, \mathrm{A}$ & $n$ \\
\hline Before thermal cycling, $I$ increasing & 76.9 & 18.5 & 75.8 & 24.6 \\
Before thermal cycling, $I$ decreasing & 76.6 & 21.0 & 75.9 & 23.2 \\
After thermal cycling, $I$ increasing & 76.8 & 19.7 & 76.2 & 21.6 \\
After thermal cycling, $I$ decreasing & 76.9 & 19.7 & 76.3 & 21.8 \\
\hline
\end{tabular}

\section{Conclusions and Future Work}

This paper discussed the design and fabrication of high temperature superconducting (HTS) rotor coils without turn-to-turn electrical insulation for NASA's 1.4 MW High Efficiency Megawatt Motor (HEMM). The characteristics of the coils, the motivation for using uninsulated coils, and important design considerations were discussed. A few sub-scale HTS coils were fabricated using a new 3D printed winding fixture. The key features of the test coils and winding fixture are described. The sub-scale coils are representative of the final design (i.e., they contain all of the important features and geometry), but require significantly less superconductor and less time to fabricate. The critical current and $\mathrm{n}$-value of two sub-scale coils were tested at $77 \mathrm{~K}$ under self field before and after a set number of aggressive thermal cycles between $77 \mathrm{~K}$ and about $5{ }^{\circ} \mathrm{C}$. Coil 1 was subjected to two thermal cycles, whereas coil 2 was subjected to four. The critical current and n-value of coil 1 remained approximately constant with no indication of thermally-induced degradation. The results for coil 2 are not conclusive, but at worst indicate only minor degradation of only the n-value.

\section{Acknowledgments}

This work was supported by the NASA Hybrid Gas-Electric Propulsion Subproject within the Advanced Air Transport Technology Project of NASA’s Aeronautics Research Mission Directorate.

\section{References}

[1] Jansen, R., Bowman, C., Jankovsky, A., Dyson, R., and Felder, J., "Overview of NASA Electrified Aircraft Propulsion (EAP) Research for Large Subsonic Transports," 53rd AIAA/SAE/ASEE Joint Propulsion Conference, AIAA Propulsion and Energy Forum, Atlanta, GA, AIAA 2017-4701, 2017. doi:10.2514/6.2017-4701.

[2] Bradley, M. K., and Droney, C. K., "Subsonic Ultra Green Aircraft Research: Phase I Final Report," NASA Contractor Report, NASA/CR-2011-216847, 2011. URL https://ntrs.nasa.gov/search.jsp? $\mathrm{R}=20110011321 \&$ hterms=NASA\%2fCR-2011-216847\&qs=N\%3D0\%26Ntk\%3DAll\%26Ntt\%3DNASA\%252FCR-2011216847\%26Ntx\%3Dmode\%2520matchallpartial

[3] Felder, J. L., Brown, G. V., DaeKim, H., and Chu, J., “Turboelectric Distributed Propulsion in a Hybrid Wing Body Aircraft," 20th International Society for Airbreathing Engines, ISABE-2011-1340, Gothenburg, Sweden, 2011. URL https://ntrs.nasa.gov/search.jsp?R=20120000856.

[4] Bradley, M. K., and Droney, C. K., "Subsonic Ultra Green Aircraft Research: Phase 2. Volume 2; Hybrid Electric Design Exploration," NASA Contractor Report, NASA/CR-2015-218704/VOL2, 2015. URL https://ntrs .nasa.gov/search. jsp?R=20150017039\&hterms=NASA\%2fCR-2015-218704\%2fVOL2\&qs= N\%3D0\%26Ntk\%3DAl1\%26Ntt\%3DNASA\%252FCR-2015-218704\%252FV0L2\%26Ntx\%3Dmode\%2520matchallpartial. 
[5] Lents, C., Hardin, L., Rheaume, J., and Kohlman, L., "Parallel Hybrid Gas-Electric Geared Turbofan Engine Conceptual Design and Benefits Analysis," 52nd AIAA/SAE/ASEE Joint Propulsion Conference, AIAA Propulsion and Energy Forum, Salt Lake City, UT, AIAA 2016-4610, 2016. doi:10.2514/6.2016-4610.

[6] Schiltgen, B. T., Freeman, J. L., and Hall, D. W., "Aeropropulsive Interaction and Thermal System Integration within the ECO-150: A Turboelectric Distributed Propulsion Airliner with Conventional Electric Machines," 16th AIAA Aviation Technology, Integration, and Operations Conference, AIAA AVIATION Forum, Washington, D.C., AIAA 2016-4064, 2016. doi:10.2514/6.2016-4064.

[7] Welstead, J., and Felder, J. L., "Conceptual design of a single-aisle turboelectric commercial transport with fuselage boundary layer ingestion," 54th AIAA Aerospace Sciences Meeting, AIAA SciTech Forum, San Diego, CA, AIAA 2016-1027, 2016. doi:10.2514/6.2016-1027.

[8] Perullo, C., Trawick, D., Armstrong, M., Tai, J. C., and Mavris, D. N., "Cycle Selection and Sizing of a Single-Aisle Transport with the Electrically Variable Engine(TM) (EVE) for Fleet Level Fuel Optimization," 55th AIAA Aerospace Sciences Meeting, AIAA SciTech Forum, Grapevine, TX, AIAA 2017-1923, 2017. doi:10.2514/6.2017-1923.

[9] Jansen, R., De Jesus-Arce, Y., Kascak, P., Dyson, R., Woodworth, A., Scheidler, J., Edwards, R., Stalcup, E., and Wilhite, J., "High Efficiency Megawatt Motor Conceptual Design," Proc. of AIAA/IEEE Propulsion and Energy Forum, Cincinnati, OH, 2018.

[10] Scheidler, J., "Preliminary Design of the Superconducting Rotor for NASA's High-Efficiency Megawatt Motor," Proc. of AIAA/IEEE Propulsion and Energy Forum, Cincinnati, OH, 2018.

[11] Wang, X., Trociewitz, U., and Schwartz, J., "Near-adiabatic quench experiments on short $\mathrm{YBa}_{2} \mathrm{Cu}_{3} \mathrm{O}_{7-\delta}$ coated conductors," Journal of Applied Physics, Vol. 101, No. 5, 2007, p. 053904. doi:10.1063/1.2435804.

[12] Yanagisawa, Y., Okuyama, E., Nakagome, H., Takematsu, T., Takao, T., Hamada, M., Matsumoto, S., Kiyoshi, T., Takizawa, A., Takahashi, M., et al., "The mechanism of thermal runaway due to continuous local disturbances in the YBCO-coated conductor coil winding," Superconductor Science and Technology, Vol. 25, No. 7, 2012, p. 075014. doi:10.1088/0953-2048/25/7/075014.

[13] Hahn, S., Park, D. K., Bascuñán, J., and Iwasa, Y., "HTS pancake coils without turn-to-turn insulation," IEEE Transactions on Applied Superconductivity, Vol. 21, No. 3, 2011, pp. 1592-1595. doi:10.1109/TASC.2010.2093492.

[14] Lécrevisse, T., and Iwasa, Y., “A (RE)BCO pancake winding with metal-as-insulation,” IEEE Transactions on Applied Superconductivity, Vol. 26, No. 3, 4700405, 2016, pp. 1-5. doi:10.1109/TASC.2016.2522638.

[15] Kim, J., Yoon, S., Cheon, K., Shin, K. H., Hahn, S., Kim, D. L., Lee, S., Lee, H., and Moon, S.-H., "Effect of resistive metal cladding of HTS tape on the characteristic of no-insulation coil," IEEE Transactions on Applied Superconductivity, Vol. 26, No. 4, 4601906, 2016, pp. 1-6. doi:10.1109/TASC.2016.2541687.

[16] Shin, H.-J., Kim, K., Choi, Y., Kwon, O., Hahn, S., Iwasa, Y., and Lee, H., "Effects of impregnating materials on thermal and electrical stabilities of the HTS racetrack pancake coils without turn-to-turn insulation," IEEE Transactions on Applied Superconductivity, Vol. 23, No. 3, 2013, pp. 7700404-7700404. doi:10.1109/TASC.2012.2234179.

[17] Song, J.-B., Hahn, S., Lécrevisse, T., Voccio, J., Bascuñán, J., and Iwasa, Y., "Over-current quench test and self-protecting behavior of a 7 T/78 mm multi-width no-insulation REBCO magnet at $4.2 \mathrm{~K}$," Superconductor Science and Technology, Vol. 28, No. 11, 2015, p. 114001. doi:10.1088/0953-2048/28/11/114001.

[18] Zhang, Y., Hazelton, D., Knoll, A., Duval, J., Brownsey, P., Repnoy, S., Soloveichik, S., Sundaram, A., McClure, R., Majkic, G., and Selvamanickam, V., "Adhesion strength study of IBAD-MOCVD-based 2G HTS wires using a peel test," 24 th Intl. Symposium on Superconductivity, Tokyo, Japan, 2011.

[19] Barth, C., Bagrets, N., Weiss, K., Bayer, C., and Bast, T., "Degradation free epoxy impregnation of REBCO coils and cables," Superconductor Science and Technology, Vol. 26, No. 5, 2013, p. 055007. doi:10.1088/0953-2048/26/5/055007.

[20] Oomen, M., Herkert, W., Bayer, D., Kummeth, P., Nick, W., and Arndt, T., "Manufacturing and test of 2G-HTS coils for rotating machines: Challenges, conductor requirements, realization," Physica C: Superconductivity and its applications, Vol. 482, 2012, pp. 111-118. doi:10.1016/j.physc.2012.04.021.

[21] Lu, J., Han, K., Sheppard, W., Viouchkov, Y., Pickard, K., and Markiewicz, W., "Lap joint resistance of YBCO coated conductors," IEEE Transactions on Applied Superconductivity, Vol. 21, No. 3, 2011, pp. 3009-3012. doi:10.1109/TASC.2010.2091934. 
[22] Kim, Y., Bascuñán, J., Lecrevisse, T., Hahn, S., Voccio, J., Park, D., and Iwasa, Y., "YBCO and Bi2223 coils for high field LTS/HTS NMR magnets: HTS-HTS joint resistivity,” IEEE Transactions on Applied Superconductivity, Vol. 23, No. 3, 2013, pp. 6800704-6800704. doi:10.1109/TASC.2013.2243195.

[23] Wang, X., Hahn, S., Kim, Y., Bascuñán, J., Voccio, J., Lee, H., and Iwasa, Y., "Turn-to-turn contact characteristics for an equivalent circuit model of no-insulation ReBCO pancake coil," Superconductor Science and Technology, Vol. 26, No. 3, 2013, p. 035012. doi:10.1088/0953-2048/26/3/035012.

[24] Ekin, J., Experimental techniques for low-temperature measurements: cryostat design, material properties and superconductor critical-current testing, p. 291, Oxford University Press, 2006. 